\title{
Variaciones limnológicas espaciotemporales de un lago altoandino tropical al norte de Perú
}

\author{
Jesús Rascón ${ }^{1, \mathbb{\Xi}}$; Fernando Corroto 2 ; Damaris Leiva-Tafur ${ }^{1} \&$ Oscar A. Gamarra \\ TORRES
}

\author{
${ }^{1}$ Instituto de Investigación para el Desarrollo Sustentable de Ceja de Selva. Universidad Nacional Toribio Rodríguez de \\ Mendoza de Amazonas. Chachapoyas, Perú. ${ }^{2}$ Departamento de Biología. Universidad Autónoma de Madrid. Madrid, España.
}

Resumen. El lago Pomacochas se localiza en la región de los bosques montanos del norte de Perú, en un área de gran importancia agropecuaria y piscícola, actividades que representan la base de la economía local. La población establecida en sus márgenes descarga en el lago parte de las aguas residuales y sin tratamiento. El principal objetivo del estudio fue determinar la dinámica espaciotemporal de este ecosistema léntico, evaluando las condiciones limnológicas y tróficas durante los períodos seco y lluvioso, y la relación entre ellas. En 15 estaciones de muestreo se analizaron las variables limnológicas transparencia, clorofila 'a', temperatura, $\mathrm{pH}$, conductividad, saturación de oxígeno, turbidez, nitratos, nitritos, amonio y fósforo reactivo soluble. Además, se calcularon los índices de estado trófico de Carlson (IET $\mathrm{C}_{\mathrm{C}}$, de estado trófico modificado de Aizaki et al. (IET ), de estado trófico modificado de Toledo et al. $\left(\right.$ IET $_{\mathrm{T}}$ ) y de estado trófico de Vollenweider et al. (TRIX). El lago mostró una variación temporal pero no espacial, tanto para las variables limnológicas como para los índices tróficos. También se analizó la dinámica de las variables limnológicas en los diferentes períodos climáticos. Los índices $\mathrm{IET}_{\mathrm{C}}$ y $\mathrm{IET}_{\mathrm{A}}$ permitieron caracterizar al lago como $\alpha$-mesotrófico a $\beta$-eutrófico, mientras que el IET como $\alpha$-mesotrófico a $\alpha$-eutrófico. El TRIX indicó que el sistema presenta una mala calidad del agua, altamente productiva, y un nivel trófico muy alto. Los resultados obtenidos revelan que el IET $_{\mathrm{A}}$ describió mejor el estado trófico del lago, mientras que el $\mathrm{IET}_{C}$ fue el que peor lo hizo. Todos estos resultados fueron estadísticamente significativos $(P<0.05)$. Los índices tróficos evidencian que el lago Pomacochas presenta una fuerte tendencia hacia la eutrofia debido a las actividades agropecuarias. Este estudio también evidenció que el sistema se encuentra condicionado por la estacionalidad climática.

[Palabras clave: eutrofización, clorofila a, índice trófico, categoría trófica, Andes]

Abstract. Spatial and temporal dynamics of the trophic state in a tropical high Andean lake in northern Perú. Lake Pomacochas is located in the montane forest region of northern Perú, in an area of great agricultural and fish farming importance, activities that represent the basis of the local economy. The population settled on its margins discharges part of its wastewater into the lake without treatment. The main aim of this study was to determine the spatio-temporal dynamics of this lentic ecosystem, evaluating the limnological and trophic conditions during the dry and rainy periods, and their relationship with each other. The following limnological variables were analyzed at 15 sampling sites: transparency, chlorophyll ' $\mathrm{a}$ ', temperature, $\mathrm{pH}$, conductivity, oxygen saturation, turbidity, nitrates, nitrites, ammonium and soluble reactive phosphorus. Besides, the following trophic indices were calculated: Carlson's trophic status index (TSI), trophic status index modified by Aizaki et al. $\left(\mathrm{TSI}_{\mathrm{A}}\right)$, trophic status index modified by Toledo et al. $\left(\mathrm{TSI}_{\mathrm{T}}\right)$ and trophic status index by Vollenweider et al. (TRIX). The lake showed temporal, but not spatial, variation for both limnological variables and trophic indices. The dynamics of the limnological variables in the different climatic periods was also analyzed. The TSI ${ }_{C}$ and TSI ${ }_{A}$ indexes indicated that the lake can be characterized as $\alpha$-mesotrophic to $\beta$ eutrophic, and according to TSI index as $\alpha$-mesotrophic to $\alpha$-eutrophic. The TRIX showed the system has a poor water quality, highly productive, and a very high trophic level. The results reveal that the $\mathrm{TSI}_{\mathrm{A}}$ best described the trophic state of the lake, while the $\mathrm{TSI}_{\mathrm{C}}$ was the worst. All these results were statistically significant $(P<0.05)$. The trophic indices evidenced that lake Pomacochas shows a strong tendency to the eutrophication due to agricultural activities. The study also showed that the system is conditioned by climatic seasonality.

[Keywords: eutrophication, chlorophyll a, trophic index, trophic category, Andes]

Editora asociada: Irina Izaguirre 


\section{INTRODUCCIÓN}

Los lagos altoandinos son uno de los recursos más frágiles y vulnerables a la contaminación (Dodds and Whiles 2010). En particular, los lagos altoandinos tropicales son ecosistemas acuáticos con características especiales, de los cuales existen pocos estudios (Rull et al. 2008; Michelutti et al. 2016; Aranguren-Riaño et al. 2018). Principalmente, se localizan en Colombia, Ecuador y norte de Perú, entre los 2000 y 4000 m s. n. m. La dinámica de estos lagos tiene grandes diferencias con los lagos localizados en las zonas bajas tropicales debido a la altitud y a las condiciones climáticas dominantes (Casallas and Gunkel 2001; Gunkel 2003; López-Martínez et al. 2017).

Uno de los problemas más frecuentes en estos ecosistemas es la eutrofización, causada sobre todo por el aumento de las concentraciones de nitrógeno y fósforo (Zamparas and Zacharias 2014), que llegan a las aguas continentales por la escorrentía superficial y subterránea (O'Neill and Davis 2012). Este aumento es resultado de actividades antrópicas como la ganadería (Zheng et al. 2013) y la agricultura (Anderson et al. 2014). Los cambios del estado trófico producen alteraciones en la composición y la abundancia de las especies de las comunidades que los habitan (Fernández et al. 2012; Naeher et al. 2012). Por una parte, hay un aumento de los productores primarios (Schoenebeck et al. 2012), en especial de las poblaciones fitoplanctónicas, que al tener una alta actividad fotosintética generan un aumento significativo de la biomasa y del oxígeno disuelto (Gao et al. 2014), en especial en las capas superiores del lago. Asimismo, aumenta la turbidez, lo que impide que la luz penetre en zonas más profundas de la columna de agua (Chen et al. 2013) y disminuye la biodiversidad (Qin et al. 2013).

El uso de índices para conocer el estado trófico de los lagos y lagunas se comenzó a desarrollar en el último cuarto del siglo XX para lagos templados en el hemisferio norte (Carlson 1977, 1980). De igual manera, se desarrollaron índices para lagos tropicales como el modificado por Toledo y por Lamparelli, ambos basados en el índice de Carlson (Silvino and Barbosa 2015). Los índices de estado trófico (IET) permiten clasificar a los lagos desde ultraoligotróficos (i.e., con niveles de nutrientes extremadamente bajos) hasta hipereutróficos (i.e., con elevadas concentraciones de nutrientes), basándose en variables fisicoquímicas y biológicas como la concentración de clorofila 'a' o la concentración de nitratos (Cony et al. 2014). Sin embargo, estos índices acarrean cierto grado de subjetividad, en particular por la falta de límites que permiten asignar la condición trófica del lago de forma precisa; por esta razón, aplicar un solo índice resulta insuficiente (Gómez et al. 2014).

El lago Pomacochas, es un lago altoandino localizado en la región de los bosques montanos del norte de Perú. Es una cuenca de gran importancia agropecuaria en la que durante los últimos años se ha favorecido la deforestación debido a fundos ganaderos o campos de cultivo. Teniendo en cuenta la importancia ecológica y económica de dicho ecosistema, el objetivo del estudio fue determinar su condición ecológica mediante la evaluación de las variables limnológicas, analizando su dinámica durante los períodos seco y lluvioso. El estado trófico del lago se evaluó aplicando cuatro índices diferentes: el índice de estado trófico de Carlson (IET ${ }_{C}$ ) (Carlson 1977), el índice de estado trófico modificado de Aizaki et al. (IET ( $_{\text {) }}$ (Aizaki et al. 1981), el índice de estado trófico modificado de Toledo et al. (IET ${ }_{\mathrm{T}}$ ) (Crossetti and Bicudo 2005) y el índice de estado trófico de Vollenweider et al. (TRIX) (Vollenweider et al. 1998). También se analizó la relación entre las variables limnológicas y estos índices, y se evaluó cuáles fueron los que mejor describieron las condiciones del sistema con el objeto su aplicación en un programa de gestión y monitoreo a largo plazo.

\section{Materiales y Métodos}

\section{Área de estudio}

El lago Pomacochas se encuentra en el distrito de Florida, provincia de Bongará (Región Amazonas) que cuenta con una población total de $\sim 6000$ personas (INEI 2018). El lago es de origen tectónico (Wetzel 2001) como consecuencia de procesos de hundimiento y levantamiento del suelo. Pertenece a la Región Hidrográfica del Amazonas, se sitúa a una altitud de 2233 m s. n. m., posee una superficie de $4.22 \mathrm{~km}^{2}$ y una profundidad máxima de $75.50 \mathrm{~m}$. Su principal aporte de agua es mediante precipitaciones y escorrentía subterránea (Barboza-Castillo et al. 2014), con una precipitación total durante todo el estudio de $1935.9 \mathrm{~mm}$; la precipitación mínima se dio en julio de 2016 (con $34.7 \mathrm{~mm}$ ) y la precipitación máxima en marzo de 
2017 (con $366.3 \mathrm{~mm}$ ). La temperatura anual promedio fue de $15.05^{\circ} \mathrm{C}$, según los datos recogidos por la estación meteorológica que el Instituto de Investigación para el Desarrollo Sustentable de Ceja de Selva tiene instalada en la zona. Sus principales afluentes son las quebradas Fichac y Congona, que atraviesan el centro urbano, mientras que su efluente denominado 'Desaguadero' se dirige hacia el río Pomacochas (Figura 1).

La actividad ganadera en la cuenca consiste en fundos con prados nativos para la explotación extensiva (Oliva et al. 2015a,b). La agricultura consiste en pequeños campos de cultivo de las principales hortalizas y verduras que se venden en el mercado local (MINAGRI 2013). Por otro lado, en el centro del lago también se ha desarrollado la acuicultura con la instalación de una pequeña piscigranja para el cultivo de trucha arcoíris (Oncorhynchus mykiss), aunque esta actividad fue abandonada en 2016. Esto ha generado que la población local pesque de forma más tradicional pejerrey (Odontesthes bonariensis) y plateados (Menidia sp.) (Barboza-Castillo et al. 2014). El lago también cuenta con dos embarcaderos, uno en la zona oeste y otro en la zona este, que sirven como medio de transporte para los agricultores y ganaderos, ya que la localidad de Pomacochas está en la costa oeste, mientras que los campos ganaderos y de cultivo se
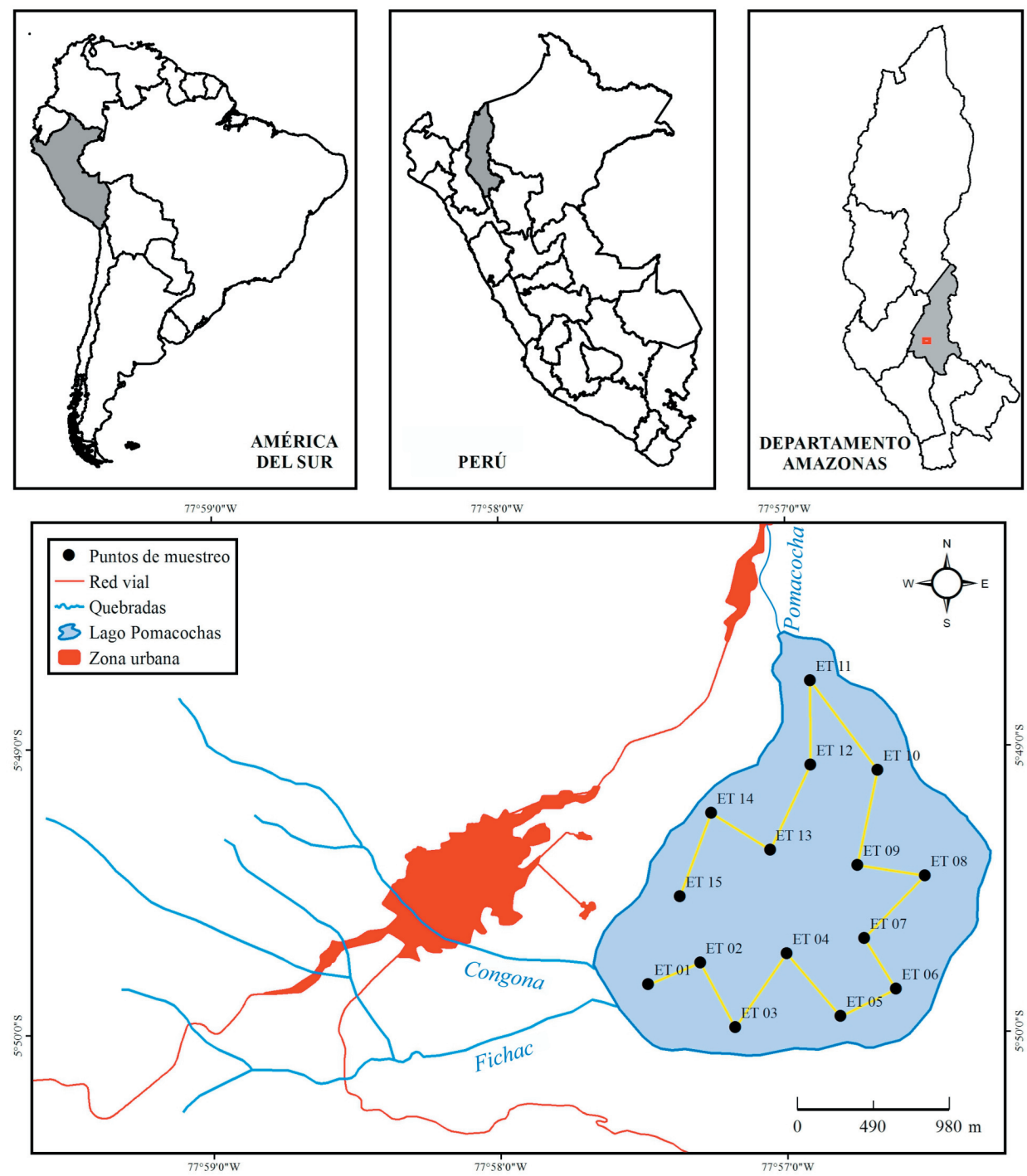

Figura 1. Ubicación de las estaciones de muestreo en el lago Pomacochas, Distrito de Florida, Provincia de Bonagrá, Región Amazonas (Perú).

Figure 1. Location of sampling stations in Lake Pomacochas, District of Florida, Province of Bongará, Amazonas Region (Perú). 
encuentran mayormente en el lado este y sur. Todas estas actividades también generan un reclamo turístico regional en la zona oeste del lago (Chávez-Ortiz et al. 2014).

\section{Metodología empleada}

Se llevaron a cabo 10 muestreos con una periodicidad mensual entre los meses de julio de 2016 y junio de 2017, cubriendo un período seco (julio a diciembre) y uno lluvioso (enero a junio); por problemas técnicos no pudieron realizarse muestreos en los meses de diciembre de 2016 y enero de 2017. Se contó en total con 10 muestreos mensuales. Se establecieron 15 puntos de muestreo a lo largo del lago siguiendo la metodología propuesta por López-Martínez and Madroñero-Palacios (2015). Se llevó a cabo un recorrido en zigzag de sur a este, de este a norte, de norte a oeste y de oeste a sur; los puntos se establecieron previamente en fase de gabinete con la ayuda del software informático ArcGIS y fueron confirmados en campo con un receptor de posicionamiento global (GPS) marca Garmin, modelo Oregon 650. La selección de las estaciones de muestreo se basó en una serie de consideraciones: ET01 y ET02 son las más cercanas a las quebradas (entradas de agua); ET04 es el punto donde se encuentra la profundidad máxima; ET03, ET05, ET06, ET07, ET08 y ET09 son las más cercanas a las zonas con producción agropecuaria; ET10 es la más próxima al embarcadero de la zona este; ET11 la más cercana al 'Desaguadero' (salida de agua); ET12 y ET13 son las más cercanas a la antigua piscigranja de truchas que se encuentra en el lago; ET15 y ET 14 son las más próximas al embarcadero de la zona oeste y al núcleo urbano.

La recolección, almacenamiento y traslado de las muestras, así como los análisis de laboratorio, se realizaron de acuerdo con APHA, AWWA y WEF (2012) y MAGRAMA $(2013 a, b)$. Las muestras para el análisis de clorofila 'a' fueron tomadas a tres profundidades en cada punto de muestreo: a nivel superficial, a un metro y en el límite de la zona fótica. Dichas muestras fueron recolectadas con una botella Van Dorn y homogenizadas en el momento. Para la determinación de clorofila 'a', las muestras de agua fueron procesadas con filtros de microfibras de vidrio Whatman GF/F de 47 $\mathrm{mm}$ de diámetro. El contenido del filtro fue utilizado para extraer la clorofila 'a', usando acetona al $90 \%$ como solvente extractor en oscuridad, de acuerdo con el procedimiento de MAGRAMA (2013b) y luego cuantificada la concentración usando la ecuación desarrollada por Jeffrey and Humphrey (1975), con ayuda de un espectrofotómetro de absorción atómica Thermo Scientific, modelo Genesys 10S UVVis. Las variables medidas in situ fueron: transparencia $(\mathrm{m})$ con un disco de Secchi; temperatura $\left({ }^{\circ} \mathrm{C}\right), \mathrm{pH}$, conductividad $(\mu \mathrm{s} / \mathrm{cm})$ y saturación de oxígeno (\%OD) con un equipo multiparamétrico marca SI Analytics, modelo HandyLab 680. Para las variables turbidez (NTU), nitratos $\left(\mathrm{N}-\mathrm{NO}_{3}^{-}\right)$, nitritos $\left(\mathrm{N}-\mathrm{NO}_{2}^{-}\right)$, amonio $\left(\mathrm{N}_{-} \mathrm{NH}_{4}^{+}\right)$y fósforo reactivo soluble (PRS), se tomaron muestras de la misma mezcla homogeneizada, refrigerándolas a $4{ }^{\circ} \mathrm{C}$ durante su traslado, para su posterior análisis en laboratorio.

La determinación del estado trófico se hizo a través de $\mathrm{IET}_{\mathrm{C}}$ (Carlson 1977), el $\mathrm{IET}_{\mathrm{A}}$ (Aizaki et

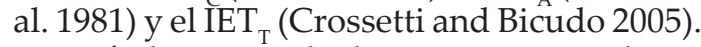
Estos índices se calcularon por separado en base a la concentración de clorofila ' $a$ ' $\left(\mathrm{mg} / \mathrm{m}^{3}\right)$, la transparencia del disco de Secchi (DS) en la columna de agua $(\mathrm{m})$ y la concentración de PRS $\left(\mathrm{mg} / \mathrm{m}^{3}\right)$. Se calculó un índice promedio con los obtenidos a partir de las tres variables. Las ecuaciones para calcular el IET $_{C}$ fueron las siguientes:

$$
\begin{gathered}
I E T_{C}(T)=10\left(6-\frac{\ln (D S)}{\ln 2}\right) \\
I E T_{C}(P R S)=10\left(6-\frac{\ln (48 / P R S)}{\ln 2}\right) \\
I E T_{C}(\text { Cloa })=10\left(6-\frac{2.04-0.68 \ln (\text { Cloa })}{\ln 2}\right) \\
I E T_{C}(\text { Promedio })=\frac{I E T_{C}(T)+I E T_{C}(P R S)+I E T_{C}(\text { Cloa })}{3}
\end{gathered}
$$

$\operatorname{IET}_{C}(\mathrm{~T})$ es el valor del índice de estado trófico de Carlson para la transparencia; IET $_{\mathrm{C}}$ (P) para el PRS; IET $_{C}$ (Cloa) para la clorofila 'a' y IET $_{C}$ (Promedio) es el valor del índice de estado trófico promedio. Las ecuaciones para calcular el $\mathrm{IET}_{\mathrm{A}}$ se detallan a continuación.

$$
\begin{gathered}
I E T_{A}(T)=10\left(2.46+\frac{3.76-1.57 \ln (D S)}{\ln 2.5}\right) \\
I E T_{A}(P R S)=10\left(2.46+\frac{6.68-1.15 \ln (P R S)}{\ln 2.5}\right) \\
I E T_{A}(\text { Cloa })=10\left(2.46+\frac{\ln (\text { Cloa })}{\ln 2.5}\right) \\
I E T_{A}(\text { Promedio })=\frac{I E T_{A}(T)+I E T_{A}(P R S)+I E T_{A}(\text { Cloa })}{3}
\end{gathered}
$$


Las ecuaciones para calcular el $\mathrm{IET}_{\mathrm{T}}$ fueron:

$$
\begin{gathered}
I E T_{T}(T)=10\left(6-\frac{0.64+\ln (D S)}{\ln 2}\right) \\
I E T_{T}(P R S)=10\left(6-\frac{(80.32 / P R S)}{\ln 2}\right) \\
I E T_{T}(\text { Cloa })=10\left(6-\frac{2.04-0.695 \times \ln (\text { Cloa })}{\ln 2}\right) \\
\left.I E T_{T} \text { (Promedio }\right)=\frac{I E T_{T}(T)+I E T_{T}(\text { PRS })+I E T_{T}(\text { Cloa })}{3}
\end{gathered}
$$

La tipificación empleada para $\operatorname{IET}_{\mathrm{C}^{\prime}} \mathrm{IET}_{\mathrm{A}} \mathrm{e}$ $\mathrm{IET}_{\mathrm{T}}$ se basó en la propuesta por ContrerasEspinosa et al. (1994) para el valor del índice promedio, al plantear una expansión en los intervalos de concentración del pigmento (Tabla 1). Por otro lado, el índice TRIX (Vollenweider et al. 1998) se basa, además de las variables anteriores, en el nitrógeno inorgánico disuelto, el fósforo reactivo soluble y la saturación de oxígeno (Tabla 2). La ecuación para calcular el TRIX fue:

$$
T R I X=\frac{\log ([\text { Cloa }] \times|\% O D| \times N I D \times P R S)+1.5}{1.2}
$$

[Cloa] es el valor de concentración de clorofila 'a' en $\mu \mathrm{g} / \mathrm{L}$; $\mid \% \mathrm{OD}$ | es el valor absoluto de la desviación del porcentaje de saturación de oxígeno disuelto (100-\%OD); NID es el nitrógeno inorgánico disuelto en $\mu \mathrm{g} / \mathrm{L}$, obtenido a partir de la suma de los nitratos $\left(\mathrm{N}-\mathrm{NO}_{3}^{-}\right)$, nitritos $\left(\mathrm{N}-\mathrm{NO}_{2}^{-}\right)$, amonio $\left(\mathrm{N}-\mathrm{NH}_{4}^{+}\right)$; PRS es el fósforo reactivo soluble en $\mu \mathrm{g} / \mathrm{L}$.

\section{Análisis de los datos}

Se aplicó un análisis de componentes principales (ACP) para simplificar y

Tabla 1. Clasificación de las categorías tróficas, según Contreras-Espinosa et al. (1994) para los valores promedio.

Table 1. Classification of the trophic categories according to Contreras-Espinosa et al. (1994) for average values.

\begin{tabular}{cc}
\hline Clasificación trófica & Valor índice \\
\hline Ultraoligotrófico & $<9$ \\
$\alpha$ oligotrófico & $10-19$ \\
$\beta$ oligotrófico & $20-29$ \\
$\gamma$ oligotrófico & $30-39$ \\
$\alpha$ mesotrófico & $40-49$ \\
$\beta$ mesotrófico & $50-59$ \\
$\alpha$ eutrófico & $60-69$ \\
$\beta$ eutrófico & $70-79$ \\
$\gamma$ eutrófico & $80-89$ \\
Hipereutrófico & $>90$ \\
\hline
\end{tabular}

Tabla 2. Clasificación de la eutrofización y calidad agua, según Vollenweider et al. (1998).

Table 2. Classification of eutrophication and water quality, according to Vollenweider et al. (1998).

\begin{tabular}{ccc}
\hline Nivel de eutrofización Calidad de agua & Valor TRIX \\
\hline Baja & Alta & $<4$ \\
Media & Buena & $4-5$ \\
Alta & Pobre & $5-6$ \\
Muy alta & Mala & $>6$ \\
\hline
\end{tabular}

ordenar las variables limnológicas. En los conglomerados obtenidos en la ACP se usó la trasformación $\ln (\mathrm{x}+1)$ para determinar diferencias espaciotemporales con un análisis de varianza multivariado (MANOVA), que se complementóconlos promediosy desviaciones estándar de las variables limnológicas para los períodos seco y lluvioso. Para el estado trófico, se calculó el valor de los diferentes índices en cada mes. Además, se utilizó un análisis Kruskal-Wallis para determinar diferencias significativas entre los índices, comparando períodos climáticos y estaciones de muestreo. Luego, se aplicó una correlación de Spearman, para analizar la relación entre los valores de los índices con las variables no usadas para su cálculo. Por último, se realizó una regresión lineal múltiple por el método por pasos de cada índice, en la que las variables dependientes corresponden a cada uno de los índices y las variables independientes en cada caso corresponden a las variables limnológicas usadas para el cálculo de cada índice. Con ello se determinó qué índice explicó mejor la condición trófica del lago Pomacochas. Todos los análisis estadísticos se realizaron a un nivel de significancia de $P<0.05$ con el software IBM SPSS v. 24.

\section{Resultados}

\section{Dinámica espaciotemporal de las variables limnológicas}

Seseleccionaron trescomponentes principales (CP) del ACP que en total explicaron el 99.88 de la varianza total de los datos (Tabla 3). El $\mathrm{CP} 1$ se correlacionó fuertemente con $\mathrm{N}-\mathrm{NO}_{2}$, clorofila y turbidez, y de forma moderada con la conductividad, el $\mathrm{pH}$, la transparencia, la saturación de oxígeno y PRS. Por otro lado, el CP2 se correlacionó fuertemente con el PRS y de forma moderada con la saturación de oxígeno y la temperatura; el CP3 mostró una elevada correlación con los nitratos y moderada con el amonio. Tanto el CP1 como el CP2 presentaron valores positivos para las variables de $\mathrm{N}^{-\mathrm{NO}_{3}}$, clorofila, turbidez, $\mathrm{pH}$, 
Tabla 3. Resultados del análisis de componentes principales de las variables limnológicas (DS: transparencia evaluada a través del disco de Secchi; Clo a: clorofila 'a'; TEMP: temperatura; $\mathrm{pH}$; $\mathrm{CE}$ : conductividad; \%OD: porcentaje de saturación

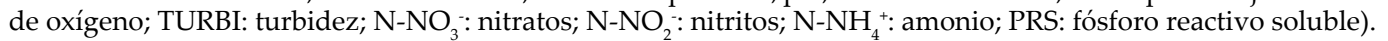

Table 3. Results of the principal component analysis of the limnological variables (DS: transparency evaluated by Secchi disk; Clo a: chlorophyll a; TEMP: temperature; $\mathrm{pH}$; CE: conductivity; \%DO: percentage of oxygen saturation; TURBI:

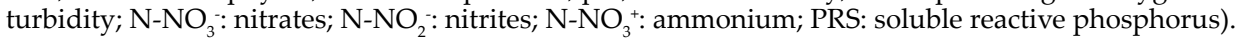

\begin{tabular}{|c|c|c|c|}
\hline & $\mathrm{CP} 1$ & $\mathrm{CP} 2$ & $\mathrm{CP} 3$ \\
\hline Total & 882049.28 & 56427.36 & 3248.66 \\
\hline Varianza (\%) & 93.55 & 5.99 & 0.34 \\
\hline V. acumulada (\%) & 93.55 & 99.54 & 99.88 \\
\hline Cargas & 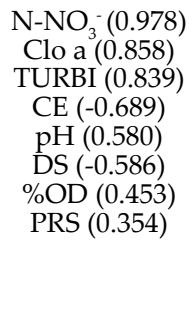 & $\begin{array}{c}\% \text { OD }(0.450) \\
\text { PRS }(0.932) \\
\text { TEMP }(-0.282)\end{array}$ & $\begin{array}{l}\mathrm{N}-\mathrm{NO}_{2}^{-}(0.997) \\
\mathrm{N}^{-} \mathrm{NH}_{4}^{+}{ }^{+}(0.392)\end{array}$ \\
\hline
\end{tabular}

Método de rotación: Varimax con normalización Kaiser a

a. La rotación ha convergido en 5 iteraciones.

saturación de oxígeno y PRS, mientras que las variables de temperatura, $\mathrm{N}-\mathrm{NO}_{2}, \mathrm{~N}-\mathrm{NH}_{4}^{+}$, transparencia y conductividad, mostraron un comportamiento negativo (Figura 2).

El análisis MANOVA de las variables limnológicas indica que existen diferencias significativas entre los períodos climáticos analizados $\left(\lambda\right.$ Wilks $=0.518, F_{3,19}=5.901, P$ $=0.005)$, registrándose los valores más altos en el período lluvioso (Tabla 4). Sin embargo, no se encontraron diferencias significativas entre las estaciones de muestreo $(\lambda$ Wilks $=0.594$,
$\left.F_{9,46}=1.232, \quad P=0.299\right)$ ni interacciones significativas (períodos $\mathrm{x}$ estaciones) $(\lambda$ Wilks=0.778, $\left.F_{6,38}=0.847, P=0.542\right)$.

\section{Variación estacional en los índices de trofismo}

En cuanto a su dinámica, según el análisis de Kruskal-Wallis, los índices se vieron afectados de forma significativa por los períodos climáticos (IET $, \chi^{2}=29.268, P<0.001$; IET, $\chi^{2}=29.268, P<0.001 ; \mathrm{IET}_{\mathrm{T}}, \chi^{2}=29.268, P=<0.001$; TRIX, $\left.\chi^{2}=29.268, P<0.001\right)$. Sin embargo, no se observaron diferencias significativas entre

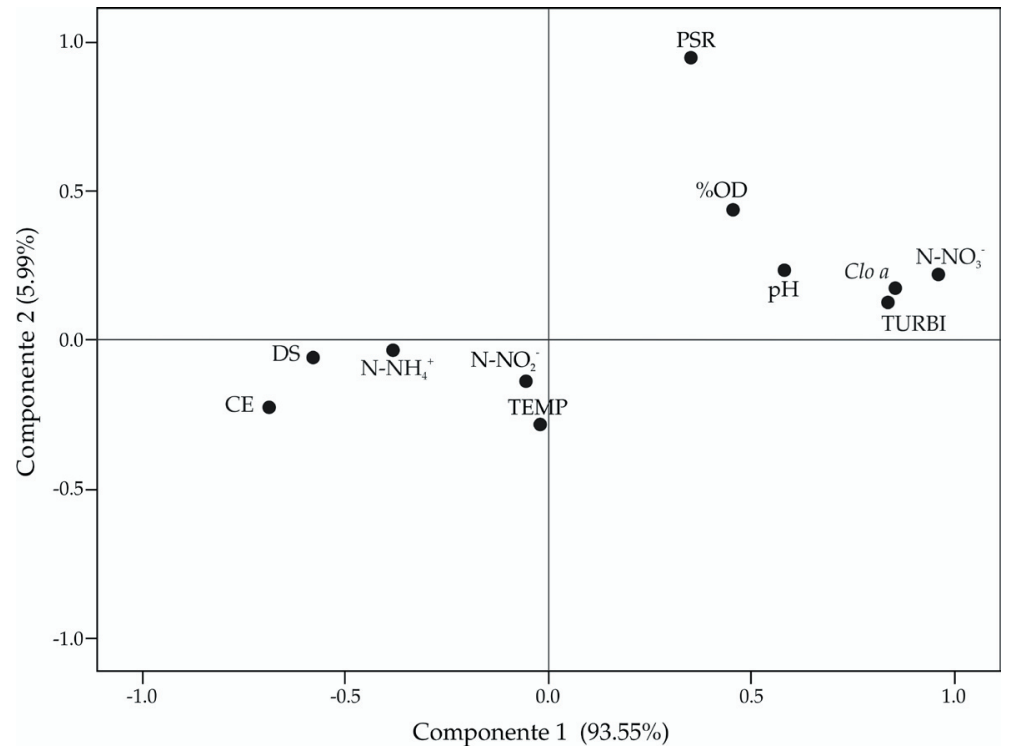

Figura 2. Gráfica de análisis de componentes principales que describe el comportamiento de las variables limnológicas (DS: transparencia; Clo a: clorofila ' $a$ '; TEMP: temperatura; $\mathrm{pH}$; CE: conductividad; \%OD: saturación de oxígeno; TURBI: turbidez; N$\mathrm{NO}_{3}^{-}$: nitratos; $\mathrm{N}-\mathrm{NO}_{2}^{-}$: nitritos; $\mathrm{N}-\mathrm{NH}_{4}^{+}$: amonio; PRS: fósforo reactivo Soluble).

Figure 2. Graph of the principal component analysis that describes the behavior of limnological variables (DS: transparency; Clo a: chlorophyll a; TEMP: temperature; $\mathrm{pH}$; CE: conductivity; \%DO: oxygen saturation; TURBI:

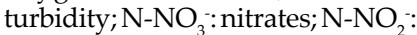
nitrites; $\mathrm{N}_{-} \mathrm{NO}_{3}^{+}$: ammonium; PRS: soluble reactive phosphorus). 
Tabla 4. Valores promedio y desviación estándar de las variables limnológicas (DS: transparencia evaluada a través del disco de Secchi; Clo a: clorofila 'a'; TEMP: temperatura; $\mathrm{pH}$; CE: conductividad; \%OD: porcentaje de saturación de

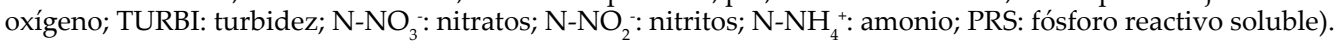

Table 4. Mean values and standard deviation of limnological variables (DS: transparency evaluated by Secchi disk; Clo a: chlorophyll a; TEMP: temperature; $\mathrm{pH}$; $\mathrm{CE}$ : conductivity; \%DO: percentage of oxygen saturation; TURBI: turbidity; $\mathrm{N}-\mathrm{NO}_{3}^{-}$: nitrates; ${\mathrm{N}-\mathrm{NO}_{2}}_{2}^{-}$: nitrites; $\mathrm{N}-\mathrm{NO}_{3}^{+}$: ammonium; PRS: soluble reactive phosphorus).

\begin{tabular}{ccccc}
\hline \multirow{2}{*}{ Variables limnológicas } & \multicolumn{2}{c}{ Período seco } & \multicolumn{2}{c}{ Período lluvioso } \\
DS & Promedio & Desviación estándar & Promedio & Desviación estándar \\
\hline Clo a & 2.14 & 0.36 & 0.74 & 0.23 \\
pH & 12.51 & 5.82 & 59.38 & 24.93 \\
TEMP & 8.47 & 0.56 & 8.47 & 0.22 \\
CE & 20.04 & 4.23 & 20.79 & 1.21 \\
$\%$ OD & 239.80 & 2.31 & 232.45 & 13.54 \\
TURBI & 114.39 & 8.89 & 116.24 & 38.40 \\
$\mathrm{~N}^{-N O}{ }_{3}^{-}$ & 1.80 & 0.34 & 13.58 & 8.64 \\
$\mathrm{~N}_{-}^{-} \mathrm{NO}_{2}^{-}$ & 1395.67 & 414.65 & 2427.45 & 1011.65 \\
$\mathrm{~N}_{-}^{-}$ & 35.04 & 81.04 & 22.45 & 5.06 \\
$\mathrm{PRS}_{4}^{+}$ & 53.62 & 19.71 & 20.20 & 2.14 \\
\end{tabular}

las estaciones de muestreo ( $\operatorname{IET}_{C}, \chi^{2}=0.089$, $P=0.993 ; \mathrm{IET}_{\mathrm{A}}, \chi^{2}=0.070, P=0.995 ; \mathrm{IET}_{\mathrm{T}}, \chi^{2}=0.085$, $P=0.995$; TRIX, $\left.\chi^{2}=0.284, P=0.963\right)$.

Los cuatro IET aplicados en el lago Pomacochas indican que el estado trófico ha evolucionado a un nivel alto de eutrofización. El IET $_{C}$ presentó un valor mínimo medio de 49.13 en septiembre de 2016, coincidiendo con el período seco, mientras que su valor máximo medio fue de 73.34 en junio de 2017,
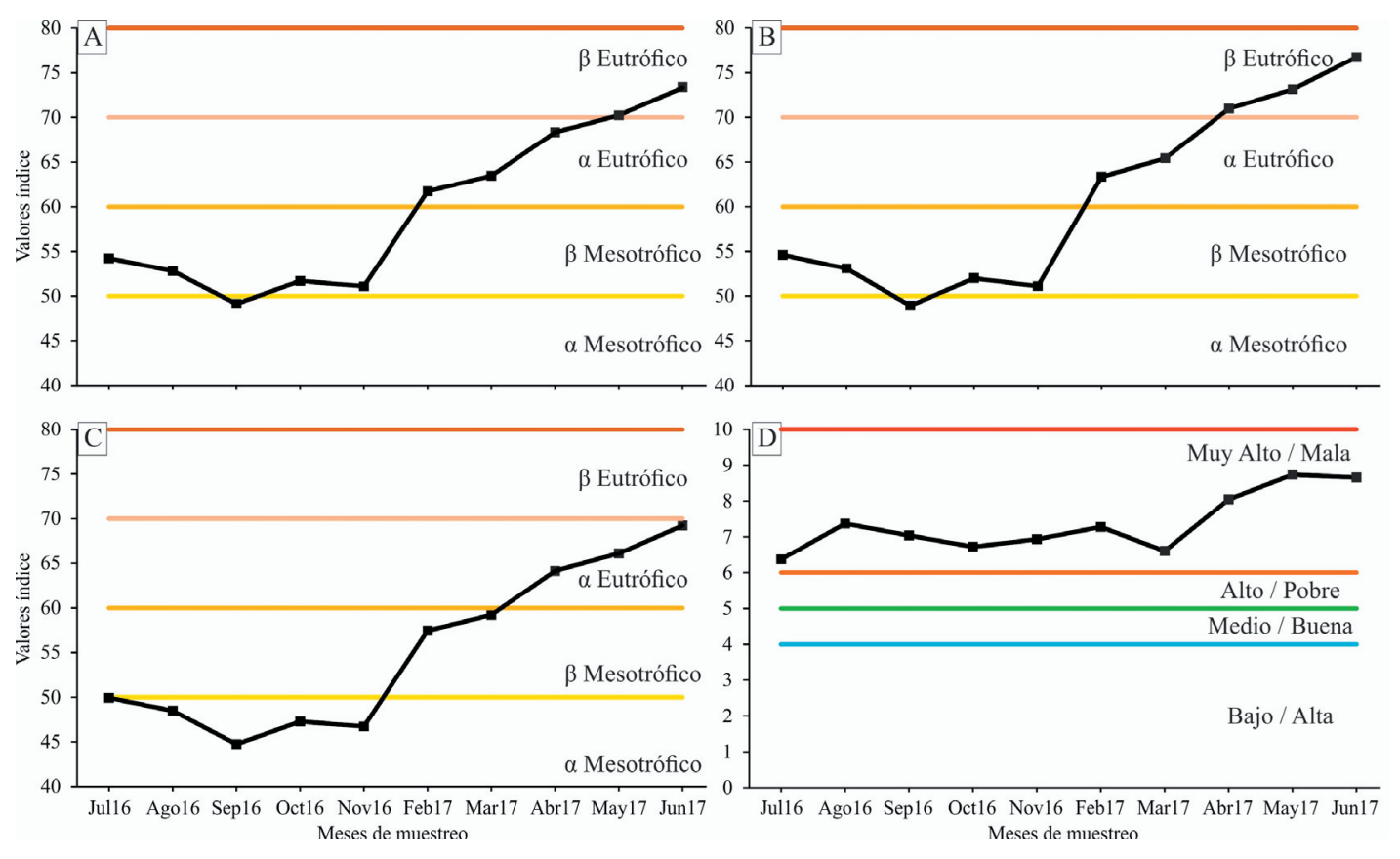

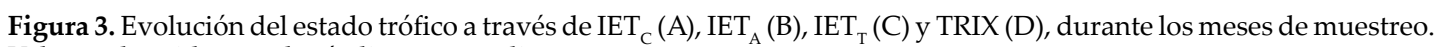
Valores obtenidos con los índices promedios.

Figure 3. Evolution of the trophic state through $\operatorname{TSI}_{C}(A), \operatorname{TSI}_{A}(B), \operatorname{TSI}_{T}(C)$ and TRIX $(D)$, during the sampling months. Values obtained with the average indices.

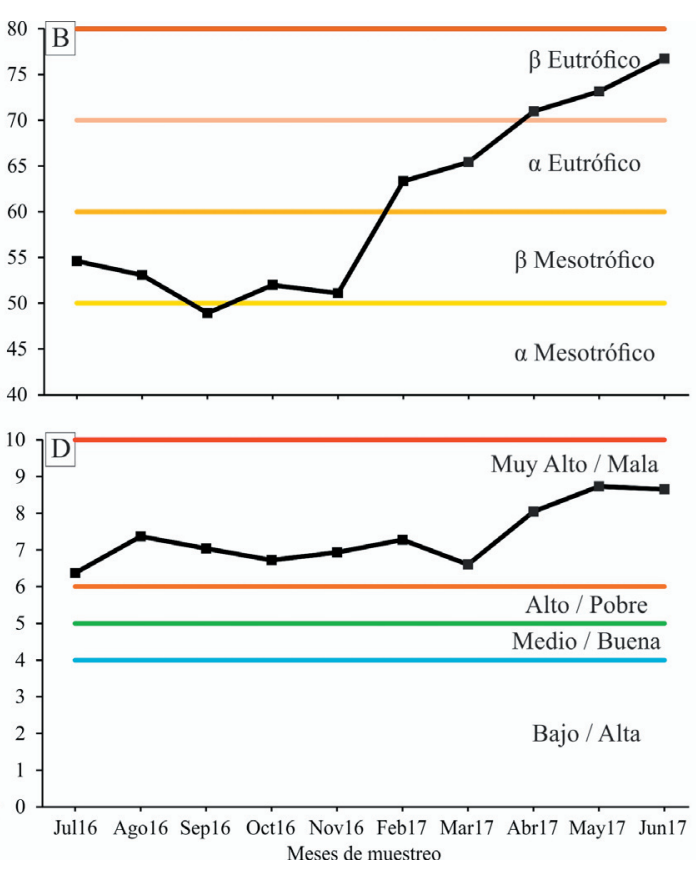

que corresponde al final del período lluvioso (Figura 3A y Tabla 5). El IET indicó un valor mínimo medio de 48.92 en septiembre de 2016 y un valor máximo medio de 76.75 en junio de 2017 (Figura 3B y Tabla 5). Por otro lado, el $\mathrm{IET}_{\mathrm{T}}$ arrojó un valor mínimo medio de 44.74 en septiembre de 2016 y un valor máximo medio de 69.22 en junio de 2017 (Figura 3C y Tabla 5). Finalmente, el índice TRIX mostró un valor mínimo medio de 6.37 en Julio de 2016 al inicio del período seco y un valor máximo 
Tabla 5. Resultados de los cuatro índices de trofismo aplicados en los diferentes meses de muestreo y en las diferentes estaciones de muestreo.

Table 5. Results of the four indices of trophism applied in the different sampling months and at the different sampling stations.

\begin{tabular}{|c|c|c|c|c|c|c|c|c|c|c|c|}
\hline Índice & Est. & Jul16 & Ago16 & Sep16 & Oct16 & Nov16 & Feb17 & Mar17 & Abr17 & May17 & Jun17 \\
\hline \multirow[t]{16}{*}{$\mathrm{IET}_{\mathrm{C}}$} & ET01 & 55.90 & 51.80 & 49.20 & 51.64 & 51.61 & 62.16 & 64.24 & 67.74 & 69.42 & 72.57 \\
\hline & ET02 & 54.70 & 53.91 & 49.56 & 50.35 & 50.60 & 62.19 & 62.29 & 69.43 & 69.84 & 73.48 \\
\hline & ET03 & 52.84 & 52.76 & 49.32 & 52.26 & 48.71 & 62.04 & 62.53 & 68.72 & 68.65 & 73.67 \\
\hline & ET04 & 53.84 & 51.64 & 50.71 & 53.01 & 50.88 & 61.39 & 62.79 & 69.08 & 68.35 & 71.91 \\
\hline & ET05 & 56.69 & 51.32 & 48.64 & 53.05 & 49.66 & 62.38 & 62.49 & 68.75 & 69.52 & 73.05 \\
\hline & ET06 & 53.76 & 54.58 & 49.26 & 52.20 & 49.38 & 63.17 & 63.66 & 68.93 & 70.84 & 73.08 \\
\hline & ET07 & 52.83 & 53.09 & 49.36 & 50.40 & 51.88 & 62.11 & 63.36 & 67.53 & 71.31 & 72.41 \\
\hline & ET08 & 52.81 & 53.73 & 49.72 & 52.99 & 51.50 & 62.46 & 64.45 & 67.75 & 68.23 & 73.52 \\
\hline & ET09 & 54.14 & 53.59 & 49.57 & 51.20 & 52.68 & 63.02 & 64.32 & 67.43 & 69.99 & 75.23 \\
\hline & ET10 & 53.90 & 51.69 & 49.33 & 52.32 & 51.20 & 60.61 & 64.48 & 66.95 & 69.30 & 74.35 \\
\hline & ET11 & 52.95 & 52.35 & 47.66 & 52.37 & 51.30 & 59.43 & 63.88 & 65.80 & 72.20 & 73.28 \\
\hline & ET12 & 54.91 & 53.15 & 46.66 & 52.13 & 51.60 & 60.47 & 63.88 & 67.58 & 71.58 & 73.55 \\
\hline & ET13 & 55.08 & 52.09 & 48.83 & 52.24 & 51.88 & 61.92 & 65.00 & 69.33 & 70.52 & 73.79 \\
\hline & ET14 & 54.87 & 53.19 & 50.18 & 51.28 & 52.36 & 60.18 & 61.66 & 70.71 & 73.01 & 73.06 \\
\hline & ET15 & 54.51 & 53.27 & 48.95 & 47.87 & 50.93 & 62.10 & 62.87 & 69.14 & 70.93 & 73.19 \\
\hline & Media & 54.24 & 52.81 & 49.13 & 51.69 & 51.08 & 61.71 & 63.46 & 68.33 & 70.24 & 73.34 \\
\hline \multirow[t]{16}{*}{$\mathrm{IET}_{\mathrm{T}}$} & ET01 & 51.64 & 47.43 & 44.81 & 47.26 & 47.27 & 57.92 & 60.02 & 63.57 & 65.27 & 68.45 \\
\hline & ET02 & 50.43 & 49.59 & 45.18 & 45.93 & 46.27 & 57.95 & 58.04 & 65.27 & 65.68 & 69.35 \\
\hline & ET03 & 48.52 & 48.44 & 44.95 & 47.90 & 44.34 & 57.80 & 58.28 & 64.55 & 64.49 & 69.54 \\
\hline & ET04 & 49.55 & 47.28 & 46.33 & 48.62 & 46.53 & 57.15 & 58.55 & 64.91 & 64.19 & 67.79 \\
\hline & ET05 & 52.47 & 46.97 & 44.24 & 48.66 & 45.29 & 58.16 & 58.25 & 64.57 & 65.38 & 68.93 \\
\hline & ET06 & 49.48 & 50.28 & 44.89 & 47.80 & 45.01 & 58.96 & 59.41 & 64.76 & 66.69 & 68.95 \\
\hline & ET07 & 48.51 & 48.78 & 44.98 & 45.96 & 47.54 & 57.88 & 59.12 & 63.36 & 67.16 & 68.29 \\
\hline & ET08 & 48.51 & 49.40 & 45.35 & 48.58 & 47.15 & 58.22 & 60.22 & 63.56 & 64.10 & 69.41 \\
\hline & ET09 & 49.86 & 49.26 & 45.19 & 46.77 & 48.35 & 58.79 & 60.06 & 63.24 & 65.85 & 71.11 \\
\hline & ET10 & 49.51 & 47.35 & 44.93 & 47.92 & 46.84 & 56.34 & 60.24 & 62.77 & 65.17 & 70.23 \\
\hline & ET11 & 48.64 & 48.02 & 43.25 & 47.99 & 46.95 & 55.16 & 59.63 & 61.59 & 68.05 & 69.16 \\
\hline & ET12 & 50.64 & 48.82 & 42.20 & 47.74 & 47.25 & 56.21 & 59.63 & 63.39 & 67.45 & 69.43 \\
\hline & ET13 & 50.81 & 47.73 & 44.42 & 47.84 & 47.53 & 57.67 & 60.77 & 65.16 & 66.38 & 69.66 \\
\hline & ET14 & 50.59 & 48.86 & 45.80 & 46.87 & 48.03 & 55.90 & 57.40 & 66.55 & 68.88 & 68.94 \\
\hline & ET15 & 50.22 & 48.97 & 44.54 & 43.39 & 46.59 & 57.85 & 58.64 & 64.97 & 66.79 & 69.07 \\
\hline & Media & 49.96 & 48.48 & 44.74 & 47.28 & 46.73 & 57.46 & 59.22 & 64.15 & 66.10 & 69.22 \\
\hline \multirow[t]{16}{*}{$\mathrm{IET}_{\mathrm{A}}$} & ET01 & 56.53 & 52.00 & 48.97 & 51.84 & 51.70 & 63.89 & 66.29 & 70.28 & 72.20 & 75.82 \\
\hline & ET02 & 55.11 & 54.34 & 49.38 & 50.48 & 50.48 & 63.88 & 64.06 & 72.26 & 72.71 & 76.95 \\
\hline & ET03 & 53.08 & 52.98 & 49.07 & 52.51 & 48.36 & 63.73 & 64.34 & 71.40 & 71.30 & 77.15 \\
\hline & ET04 & 54.15 & 51.81 & 50.73 & 53.49 & 50.88 & 62.97 & 64.64 & 71.87 & 70.93 & 75.07 \\
\hline & ET05 & 57.32 & 51.38 & 48.37 & 53.57 & 49.48 & 64.08 & 64.27 & 71.50 & 72.27 & 76.41 \\
\hline & ET06 & 54.06 & 55.10 & 49.00 & 52.60 & 49.16 & 64.96 & 65.68 & 71.68 & 73.87 & 76.44 \\
\hline & ET07 & 53.04 & 53.35 & 49.17 & 50.57 & 52.01 & 63.78 & 65.30 & 70.03 & 74.41 & 75.65 \\
\hline & ET08 & 52.98 & 54.16 & 49.57 & 53.54 & 51.57 & 64.22 & 66.56 & 70.34 & 70.74 & 76.93 \\
\hline & ET09 & 54.48 & 53.98 & 49.40 & 51.51 & 52.94 & 64.84 & 66.48 & 69.97 & 72.83 & 78.96 \\
\hline & ET10 & 54.12 & 51.78 & 49.17 & 52.73 & 51.24 & 62.14 & 66.63 & 69.38 & 71.99 & 77.91 \\
\hline & ET11 & 53.18 & 52.53 & 47.26 & 52.71 & 51.36 & 60.76 & 65.92 & 68.08 & 75.49 & 76.67 \\
\hline & ET12 & 55.37 & 53.46 & 46.20 & 52.48 & 51.70 & 61.95 & 65.94 & 70.12 & 74.70 & 76.99 \\
\hline & ET13 & 55.58 & 52.31 & 48.63 & 52.66 & 52.07 & 63.61 & 67.25 & 72.15 & 73.46 & 77.29 \\
\hline & ET14 & 55.35 & 53.53 & 50.11 & 51.54 & 52.57 & 61.66 & 63.35 & 73.75 & 76.36 & 76.43 \\
\hline & ET15 & 54.97 & 53.55 & 48.78 & 47.71 & 50.90 & 63.81 & 64.69 & 71.91 & 73.94 & 76.57 \\
\hline & Media & 54.62 & 53.08 & 48.92 & 52.00 & 51.09 & 63.35 & 65.43 & 70.98 & 73.15 & 76.75 \\
\hline \multirow[t]{16}{*}{ TRIX } & ET01 & 6.71 & 6.08 & 6.24 & 7.15 & 7.37 & 6.96 & 7.14 & 8.37 & 8.80 & 8.86 \\
\hline & ET02 & 6.63 & 7.49 & 6.30 & 6.53 & 7.26 & 7.11 & 6.81 & 8.34 & 8.80 & 8.69 \\
\hline & ET03 & 6.24 & 7.88 & 7.55 & 7.40 & 6.67 & 7.18 & 6.38 & 7.86 & 7.95 & 8.22 \\
\hline & ET04 & 6.63 & 7.30 & 7.50 & 7.09 & 6.68 & 7.14 & 6.45 & 7.73 & 8.37 & 8.25 \\
\hline & ET05 & 7.46 & 7.06 & 7.14 & 6.96 & 6.46 & 7.41 & 6.51 & 7.64 & 8.55 & 8.27 \\
\hline & ET06 & 6.39 & 7.36 & 7.31 & 6.73 & 6.49 & 7.80 & 6.55 & 7.96 & 8.71 & 8.50 \\
\hline & ET07 & 5.23 & 7.64 & 7.13 & 6.30 & 6.87 & 7.51 & 6.62 & 7.93 & 8.75 & 8.51 \\
\hline & ET08 & 5.37 & 7.01 & 7.22 & 6.48 & 6.95 & 7.46 & 6.77 & 7.90 & 8.79 & 8.62 \\
\hline & ET09 & 5.65 & 7.07 & 7.16 & 6.14 & 7.13 & 7.61 & 6.47 & 7.89 & 8.74 & 8.62 \\
\hline & ET10 & 6.10 & 6.90 & 6.97 & 6.55 & 6.88 & 7.08 & 6.66 & 7.97 & 8.83 & 8.62 \\
\hline & ET11 & 5.85 & 7.04 & 6.74 & 6.84 & 6.90 & 7.02 & 6.61 & 7.72 & 8.62 & 8.55 \\
\hline & ET12 & 6.56 & 7.04 & 6.24 & 6.66 & 6.96 & 7.20 & 6.55 & 7.97 & 8.84 & 8.52 \\
\hline & ET13 & 7.00 & 7.07 & 7.24 & 6.97 & 6.93 & 7.35 & 6.53 & 8.38 & 9.03 & 9.16 \\
\hline & ET14 & 6.90 & 6.93 & 7.67 & 6.91 & 7.23 & 6.94 & 6.27 & 8.55 & 9.25 & 9.18 \\
\hline & ET15 & 6.76 & 7.21 & 7.21 & 6.09 & 7.11 & 7.42 & 6.61 & 8.47 & 9.05 & 9.22 \\
\hline & Media & 6.37 & 7.14 & 7.04 & 6.72 & 6.93 & 7.28 & 6.60 & 8.05 & 8.74 & 8.65 \\
\hline
\end{tabular}


medio de 8.74 en el mes de mayo de 2017, correspondiente al período lluvioso (Figura 3D y Tabla 5).

Los valores de los IET $_{C}$ y el IET $_{\mathrm{A}}$ indican una variación en el sistema desde un estado $\beta$-mesotrófico a uno $\beta$-eutrófico, mientras que con el IET $_{\mathrm{T}}$ el estado trófico varía desde un estado $\alpha$-mesotrófico hasta uno $\alpha$-eutrófico. Por otro lado, el TRIX indicó siempre un nivel de eutrofización muy alto con una calidad de agua mala para el lago Pomacochas (Figura 3).

\section{Relación entre indices y variables limnológicas}

Los índices IET $_{\mathrm{C}^{\prime}}$ IET $_{\mathrm{A}}$ e IET $\mathrm{T}$ están muy relacionados de forma positiva con los nitratos

Tabla 6. Correlación de las variables fisicoquímicas con los índices de estado trófico (DS: transparencia; TEMP: temperatura; $\mathrm{pH}$; $\mathrm{CE}$ : conductividad; \%OD: saturación de oxígeno; TURBI: turbidez; $\mathrm{N}_{-} \mathrm{NO}_{3}^{-}$: nitratos; $\mathrm{N}^{-\mathrm{NO}_{2}}{ }^{-}$:

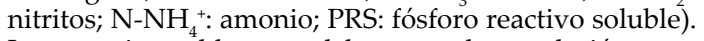
Los espacios en blanco se deben a que la correlación entre la variable y el índice no se calculó, al usarse dicha variable para el cálculo del índice.

Table 6. Correlation of physicochemical variables with trophic status indices (DS: transparency; TEMP: temperature; $\mathrm{pH}$; $\mathrm{CE}$ : conductivity; \%DO: oxygen saturation; TURBI: turbidity; $\mathrm{N}^{-\mathrm{NO}_{3}}$ : nitrates; $\mathrm{N}-\mathrm{NO}_{2}{ }_{2}^{-}$

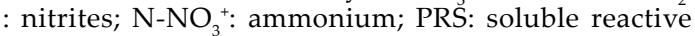
phosphorus). The blanks are because the correlation between the variable and the index was not calculated when the variable was used to calculate the index.

\begin{tabular}{ccccc}
\hline & IETC & IETA & IETT & TRIX \\
\hline \multirow{2}{*}{$\mathrm{DS}$} & & & & -0.624 \\
& & & & $0.000^{* *}$ \\
$\mathrm{pH}$ & 0.252 & 0.252 & 0.255 & 0.498 \\
& 0.116 & 0.117 & 0.112 & $0.001^{* *}$ \\
$\mathrm{TEMP}$ & -0.246 & -0.247 & -0.246 & -0.435 \\
& 0.125 & 0.124 & 0.126 & $0.005^{* *}$ \\
$\mathrm{CE}$ & -0.347 & -0.347 & -0.348 & -0.494 \\
& $0.028^{*}$ & $0.028^{*}$ & $0.028^{*}$ & $0.001^{* *}$ \\
$\% \mathrm{OD}$ & 0.110 & 0.109 & 0.108 & \\
& 0.501 & 0.502 & 0.506 & \\
$\mathrm{TURBI}$ & 0.877 & 0.876 & 0.873 & 0.656 \\
& $0.000^{* *}$ & $0.000^{* *}$ & $0.000^{* *}$ & $0.000^{* *}$ \\
$\mathrm{~N}^{*} \mathrm{NO}_{3}^{-}$ & 0.704 & 0.703 & 0.693 & \\
& $0.000^{* *}$ & $0.000^{* *}$ & $0.000^{* *}$ & \\
$\mathrm{~N}^{-} \mathrm{NO}_{2}{ }^{-}$ & 0.553 & 0.554 & 0.548 & \\
& $0.000^{* *}$ & $0.000^{* *}$ & $0.000^{* *}$ & \\
$\mathrm{~N}^{-} \mathrm{NH}_{4}{ }^{+}$ & -0.581 & -0.580 & -0.582 & \\
& $0.000^{* *}$ & $0.000^{* *}$ & $0.000^{* *}$ & \\
\hline
\end{tabular}

${ }^{*} P<0.05 ;{ }^{* *} P<0.01$ y los nitritos, y de manera negativa con el amonio. También tienen una baja relación negativa con la conductividad eléctrica. Por su parte, el índice TRIX tiene una alta relación negativa con la transparencia y positiva con la turbidez; por otro lado, tiene una moderada relación positiva con el $\mathrm{pH}$ y una moderada relación negativa con la temperatura y la conductividad. Todas las variables altamente relacionadas con los índices coinciden con las principales variables para el cálculo de estos índices (Tabla 6).

\section{Validación de índices}

En cuanto a la validación de los índices, todos muestran un $\mathrm{R}^{2}$ ajustado y un error estándar muy parecido entre ellos, siendo el IET $_{\mathrm{A}}$ el de mejor $\mathrm{R}^{2}$ y menor error, mientras que el IET $_{C}$ presenta el $\mathrm{R}^{2}$ más bajo y el error más alto (Tabla 7).

\section{Discusión}

\section{Dinámica espaciotemporal de las variables limnológicas del lago Pomacochas}

EllagodePomacochas presenta características típicas de lagos o lagunas altoandinas pertenecientes al sistema amazónico, que se destaca por precipitaciones de descarga regular y torrentoso con una estacionalidad muy marcada, diferenciándose un período seco y un período de lluvias (Ramírez et al. 2003). Algunos autores (Naeher et al. 2012; Santos et al. 2014; Harding et al. 2015) describen la dinámica del estado trófico de un lago como la sinergia de varios factores, entre los que se cuentan el alto tiempo de residencia, la deforestación y el período de muestreo. Este último factor, en el caso del lago Pomacochas, viene dado por la estacionalidad del régimen de lluvias en la zona (Rascón et al. 2020).

La carga externa de nutrientes como el nitrógeno o el fósforo aumenta en el período de lluvias (Reichwaldt and Ghadouani 2012; Eugercios-Silva et al. 2017), lo cual favorece la llegada de estos nutrientes a la masa de agua por el lixiviado y la escorrentía, sobre todo en zonas donde hay una gran deforestación (Pérez et al. 2018). Además, las actividades

Tabla 7. Resultados del modelo de regresión lineal para cada índice.

Table 7. Results of the linear regression model for each index.

\begin{tabular}{ccccc}
\hline Índice & Significancia & $\mathrm{R}^{2}$ & $\mathrm{R}^{2}$ ajustado & Error estándar \\
\hline IET $_{\mathrm{C}}$ & $0.000^{*}$ & $86.8 \%$ & $85.7 \%$ & 0.32 \\
IET $_{\mathrm{A}}$ & $0.000^{*}$ & $92.3 \%$ & $91.7 \%$ & 0.28 \\
IET $_{\mathrm{T}}$ & $0.000^{*}$ & $89.4 \%$ & $88.5 \%$ & 0.30 \\
TRIX & $0.000^{*}$ & $90.2 \%$ & $89.1 \%$ & 0.29 \\
\hline
\end{tabular}


ganaderas (Schindler et al. 2012) y agrícolas (Zhao et al. 2012) también favorecen un aumento en los niveles de clorofila ' $a$ ' en el agua, como respuesta casi inmediata a la presencia de estos nutrientes, principalmente del fósforo. Las condiciones particulares del lago, como su elevado tiempo de residencia del agua (Wetzel 2001) y una circulación del agua poco efectiva de la columna de agua, favorece la eutrofización (Aizaki et al. 1987).

\section{Dinámica y variación estacional de los índices de trofismo del lago Pomacochas}

En este trabajo se observó que todos los índices sólo presentaron diferencias temporales, lo que indica que el régimen de lluvias influye en gran media en la dinámica del estado trófico (Chaves et al. 2013). Resultados similares fueron reportados para el lago Titicaca (Archundia et al. 2017), donde se observó que las condiciones de eutrofización empeoran en el período de lluvias en las zonas relacionadas con la agricultura.

Los IET aplicados muestran ciertas diferencias en los compartimientos tróficos, sobre todo entre el $\mathrm{IET}_{\mathrm{T}}$ respecto al $\mathrm{IET}_{\mathrm{C}}$ y el $\mathrm{IET}_{\mathrm{A}}$. Esta diferencia puede deberse a que el $\operatorname{IET}_{\mathrm{T}}$ es un índice desarrollado para lagos tropicales en donde los valores de la transparencia se ven muy influenciados por la turbidez inorgánica, la cual es normal en este tipo de sistemas ya que se encuentran en las zonas bajas donde hay una gran ingreso de sedimentos; por este motivo resulta conveniente darle más importancia a los valores de clorofila 'a' que a los de transparencia (Venturoti et al. 2015). Por otro lado, el índice TRIX clasifica al lago Pomacochas con un nivel alto de eutrofización y una mala calidad de agua a lo largo de todos los meses. Este índice refleja diferencias temporales influidas por el régimen de lluvias y su relación con el lixiviado de nutrientes hacia el lago. Este índice incluye más variables que los otros, como el oxígeno disuelto, y no tiene en cuenta la transparencia, ya que se desarrolló, en un principio, para sistemas costeros (Vollenweider et al. 1998).

\section{Relación entre variables limnológicas e indices del lago Pomacochas}

Es de vital importancia establecer los rangos en las concentraciones nutrientes en el lago para caracterizar su calidad del agua considerando su estado trófico, lo que permitirá llevar a cabo una adecuada gestión desde el punto de vista ganadero, agrícola y ecológico. Las investigaciones en diversas zonas altoandinas y tropicales muestran diferencias en sus concentraciones denitrógeno y fósforo, además de diferencias en su turbidez y conductividad, y por lo tanto en su nivel trófico (Pulido-López and Pinilla-Agudelo 2017; Van Colen et al. 2017). Como se sabe, la concentración de clorofila 'a' se relaciona de forma positiva con el nitrógeno y el fósforo (Filstrup and Downing 2017), mientras que la transparencia está relacionada de forma negativa con la turbidez y la conductividad (Lagomarsino et al. 2014).

De todas las variables fisicoquímicas analizadas, la transparencia mostró una relación inversa con el índice TRIX, indicando que una disminución de esta variable conlleva a un aumento en el valor de este índice. Otra relación inversa se observó entre la variable amonio y los índices IET $_{\mathrm{C}^{\prime}}$ IET $_{\mathrm{A}}$ y IET $_{\mathrm{T}^{\prime}}$ pero ésta podría deberse al metabolismo del nitrógeno en el lago Pomacochas. Una coincidencia entre valores bajos de amonio y valores altos de estos índices puede deberse a que el nitrógeno se encuentre en forma de nitratos o en la biomasa fitoplanctónica (Yao et al. 2017; Ren et al. 2019).

También se registró una correlación negativa entre la conductividad y todos los índices, siendo más fuerte en caso del índice TRIX, lo que se debe a que es un índice desarrollado principalmente para ecosistemas marinos (Giovanardi and Vollenweider 2004; Primpas and Karydis 2011). Un comportamiento contrario se observó entre la turbidez y los índices, que mostraron una relación positiva, siendo más fuerte para los índices IET $_{C}$, IET $_{\mathrm{A}}$ $\mathrm{y} \mathrm{IET}_{\mathrm{T}}$. Esto es esperable, dada la relación negativa entre la turbidez y la transparencia evaluada a través del disco de Secchi, variable que se usó para calcular dichos índices (Lagomarsino et al. 2014).

Por otro lado, cabe mencionar que el índice TRIX fue el único que presentó una correlación con el pH y la temperatura. En ambos casos, de forma moderada, pero positiva para el pH y negativa para la temperatura. Tanto el $\mathrm{pH}$ como la temperatura son variables que afectan al oxígeno disuelto y al crecimiento de la biomasa fitoplanctónica $y$, por lo tanto, a la concentración de la clorofila 'a', variables indispensables para el cálculo del índice TRIX (El Zokm et al. 2018; Ni et al. 2018; Rahman and Hamidah 2020).

Los nitratos y nitritos mostraron una alta correlación con los índices IET $_{\mathrm{C}^{\prime}} \mathrm{IET}_{\mathrm{A}} \mathrm{y}$ 
$\mathrm{IET}_{\mathrm{T}}$, lo que era esperable, dado que estos nutrientes son necesarios para el aumento de la biomasa fitoplanctónica (Kozak et al. 2020). Cabe destacar que las actividades que se realizan alrededor del lago Pomacochas, principalmente la agropecuaria en la zona este y sur del lago, son grandes fuentes de nitrógeno y proporcionan cantidades importantes de nutrientes como nitratos y nitritos.

\section{Validación de indices en el lago Pomacochas}

Los índices IET $_{\mathrm{C}^{\prime}}$ IET $_{\mathrm{A}}$ e IET $\mathrm{IT}_{\mathrm{T}}$ se consideran técnicamente poco complicados, en parte por su facilidad para calcular las variables necesarias para avalar sus conclusiones, como son la transparencia evaluada a través del disco de Secchi o la concentración de clorofila 'a' (Crossetti and Bicudo 2005; Carlson 1977; Aizaki et al. 1981). Por el contrario, el índice TRIX, resultó menos sensible a pequeñas diferencias, calificando a la mayoría de las estaciones en un mismo nivel trófico durante los dos períodos analizados; además, es técnicamente más complicado y costoso por la cantidad de variables que se requieren para su cálculo (Vollenweider et al. 1998). Por eso, al aplicar la regresión lineal se ve que el índice IET $_{\mathrm{A}}$ se ajusta mejor a la realidad trófica del lago Pomacochas que el resto de índices, presentando incluso un menor error estándar.

\section{Conclusión}

El lago Pomacochas se encuentra en un estado de eutrofia muy avanzado (entre $\beta$-eutrófico y $\alpha$-eutrófico) debido a los desechos que generan la ganadería y la agricultura que se realizan en la zona. Estas actividades generaron una gran deforestación, lo que favorece más su impacto en el lago. Los niveles más altos de trofismo se observaron en el período lluvioso, por lo que el estado trófico depende del régimen de lluvias, ya que éstas arrastran todos los desechos nitrogenados y fosforados relacionados con las actividades agropecuarias. Este estado de eutrofia avanzando es un riesgo para posibles actividades recreativas. Asimismo, otras actividades se ven amenazadas seriamente, tales como la pesca que se practica a lo largo de todo el lago y el turismo.

La comparación entre índices de trofismo realizada en este trabajo indica que $I_{E T}$ es el que mejor se ajusta a las condiciones altoandina tropical del lago Pomacochas, ya que es el que reflejó mejor las condiciones tróficas temporales de este lago. En contraposición, el TRIX es el índice que peor reflejó estas condiciones, por lo que se puede considerar poco idóneo para su aplicación en lagos altoandinos tropicales.

Agradecimientos. Esta investigación fue realizada gracias al proyecto SNIP N 316401 "Creación de los Servicios de un Laboratorio para el manejo de Recursos Hídricos en la Universidad Nacional Toribio Rodríguez de Mendoza, región Amazonas" - RECURSOS HÍDRICOS, ejecutado por el Instituto de Investigación para el Desarrollo Sustentable de Ceja de Selva (INDES-CES) de la Universidad Nacional Toribio Rodríguez de Mendoza de Amazonas (UNTRM), el cual fue financiado por el Sistema Nacional de Inversión Pública (SNIP) del Ministerio de Economía y Finanzas (MEF) del Perú. Agradecemos la colaboración de Melanio Gupio Dett por su gran ayuda en la recolección de muestras.

\section{ReFERENCIAS}

Aizaki, M., A. Otsuki, T. Fukushima, T. Kawai, M. Hosomi, and K. Muraoka. 1981. Application of Modified Carlson's Trophic State Index to Japanese lakes and its relationships to other parameters related to trophic state. Research Report from the Nacional Institute for Enviromental Studies 23:13-31.

Aizaki, M., A. Terashima, H. Nakahara, T. Nishio, and Y. Ishida. 1987. Trophic status of Tilitso, a high altitude Himalayan lake. Hydrobiologia 153:217-24. https://doi.org/10.1007/BF00007208.

Anderson, N. J., H. Bennion, and A. F. Lotter. 2014. Lake eutrophication and its implications for organic carbon sequestration in Europe. Global Change Biology 20:2741-51. https://doi.org/10.1111/gcb.12584.

APHA, AWWA and WEF. 2017. Standard Methods for the Examination of Water and Wastewater. American Public Health Association/American Water Works Association/Water Environment Federation, Washington DC, USA.

Aranguren-Riaño, N. J., J. B. Shurin, A. Pedroza-Ramos, C. L. Muñoz-López, R. López, and O. Cely. 2018. Sources of nutrients behind recent eutrophication of Lago de Tota, a high mountain Andean lake. Aquatic Sciences 80:39. https: //doi.org/10.1007/s00027-018-0588-x.

Archundia, D., C. Duwig, L. Spadini, G. Uzu, S. Guédron, M. C. Morel, R. Cortez, O. Ramos Ramos, J. Chincheros, and J. M. F. Martins. 2017. How uncontrolled urban expansion increases the contamination of the Titicaca lake basin (El Alto, La Paz, Bolivia). Water, Air, and Soil Pollution 228:1-17. https://doi.org/10.1007/s11270-016-3217-0.

Barboza-Castillo, E., J. L. Maicelo, C. Vigo-Mestanza, J. Castro-Silupú, and M. Oliva. 2014. Análisis morfométrico y batimétrico del lago Pomacochas (Perú). INDES Revista de Investigación para el Desarrollo Sustentable 2:90-97. 
Carlson, R. E. 1977. A trophic state index for lakes. Limnology and Oceanography 22:361-69. https://doi.org/10.4319/ lo.1977.22.2.0361.

Carlson, R. E. 1980. More complications in the Chlorophyll-Secchi Disk relationship. Limnology and Oceanography 25:379-82. https://doi.org/10.4319/lo.1980.25.2.0379.

Casallas, J. E., and G. Gunkel. 2001. Algunos aspectos limnológicos de un lago altoandino: El lago San Pablo, Ecuador. Limnetica 20:215-232.

Chanamé-Zapata, F., M. Custodio, C. Poma-Chávez, and A. Huamán-De la Cruz. 2020. Nutrient concentrations and trophic state of three Andean lakes from Junín, Perú. Ambiente and Agua 15:e2525. https://doi.org/10.4136/ambiagua. 2525.

Chaves, F. I. B., P. De F. Lima, R. C. Leitão, W. D. Paulino, and S. T. Santaella. 2013. Influence of rainfall on the trophic status of a Brazilian semiarid reservoir. Acta Scientiarum - Biological Sciences 35:505-11. https://doi.org/10.4025/ actascibiolsci.v35i4.18261.

Chávez-Ortiz, J., D. Leiva-Tafur, J. Rascón, I. Hoyos, and F. Corroto. 2014. Estado trófico del lago Pomacochas a través de parámetros fisicoquímicos y bacteriológicos. INDES Revista de Investigación para el Desarrollo Sustentable 2: 70-78.

Chen, F., T. Shu, E. Jeppesen, Z. Liu, and Y. Chen. 2013. Restoration of a subtropical eutrophic shallow lake in China: Effects on nutrient concentrations and biological communities. Hydrobiologia 718:59-71. https://doi.org/10.1007/ s10750-013-1603-9.

Contreras-Espinosa, F., O. Castañeda-López, and A. García-Nagaya. 1994. La clorofila a como base para un índice trófico en lagunas costeras Mexicanas. Anales del Instituto de Ciencias del Mar y Limnología - UNAM 21:55-56.

Cony, N. L., N. C. Ferrer, and E. J. Cáceres. 2014. Evolución del estado trófico y estructura del fitoplancton de un lago somero de la Región Pampeana: Laguna Sauce Grande (Pcia. de Buenos Aires, Argentina). Biología Acuaitica 30: 79-91.

Crossetti, L. O., and C. E. M. Bicudo. 2005. Structural and functional phytoplankton responses to nutrient impoverishment in mesocosms placed in a shallow eutrophic reservoir (Garcas Pond), Sao Paulo, Brazil. Hydrobiologia 541:71-85. https://doi.org/10.1007/s10750-004-4668-7.

Dodds, W. K., and M. R. Whiles. 2010. Freshwater Ecology. Academic Press Elsevier, San Diego, California, USA. https: //doi.org/10.1016/B978-0-12-374724-2.00024-6.

El Zokm, G. M., H. R. Z. Tadros, M. A. Okbah, and G. H. Ibrahim. 2018. Eutrophication assessment using TRIX and Carlson's indices in Lake Mariout Water, Egypt. Egyptian Journal of Aquatic Biology and Fisheries 22:321-39. https: //doi.org/10.21608/ejabf.2018.23918.

Eugercios-Silva, A. R., M. Álvarez-Cobelas, and E. Monter- González. 2017. Impactos del nitrógeno agrícola en los ecosistemas acuáticos. Ecosistemas 26:37-44. https://doi.org/10.7818/ECOS.2017.26-1.06.

Filstrup, C. T., and J. A. Downing. 2017. Relationship of Chlorophyll to Phosphorus and Nitrogen in Nutrient-Rich Lakes. Inland Waters 6:155-64. https://doi.org/10.1080/20442041.2017.1375176.

Fernández, C., E. R. Parodi, and E. J. Cáceres. 2012. Phytoplankton structure and diversity in the eutrophic-hypereutrophic reservoir Paso de las Piedras, Argentina. Limnology 13:13-25. https://doi.org/10.1007/s10201-011-0347-3.

Ganguly, D., S. Patra, P. R. Muduli, K. V. Vardhan, K. R. Abhilash, R. S. Robin, and B. R. Subramanian. 2015. Influence of Nutrient Input on the Trophic State. Journal of Earth System Science 124:1005-17. https://doi.org/10.1007/s12040015-0582-9.

Gao, Y., J. M. O'Neil, D. K. Stoecker, and J. C. Cornwell. 2014. Photosynthesis and nitrogen fixation during cyanobacteria blooms in an oligohaline and Tidal freshwater estuary. Aquatic Microbial Ecology 72:127-42. https://doi.org/10.3354/ ame01692.

Giovanardi, F., and R. A. Vollenweider. 2004. Trophic Conditions of Marine Coastal Waters: Experience in Applying the Trophic Index TRIX to Two Areas of the Adriatic and Tyrrhenian Seas. Journal of Limnology 63:199-218. https: //doi.org/10.4081/jlimnol.2004.199.

Gómez, R., C. Tovilla, E. Barba, O. Castañeda, F. J. Valle, E. I. Romero, and E. Ramos. 2014. Índices tróficos de importancia ecológica y su relación con algunas variables fisico-químicas en el sistema lagunar. Revista Cubana de Investigaciones Pesqueras 31:47-57.

Gunkel, G. 2003. Limnología de un lago tropical de alta montaña, en Ecuador: Características de los sedimentos y tasa de sedimentación. Revista de Biología Tropical 51:381-90.

Harding, L. W., J. E. Adolf, M. E. Mallonee, W. D. Miller, C. L. Gallegos, E. S. Perry, J. M. Johnson, K. G. Sellner, and H. W. Paerl. 2015. Effects on phytoplankton floral composition in Chesapeake Bay. Estuarine, Coastal and Shelf Science 162:53-68. https://doi.org/10.1016/j.ecss.2014.12.030.

Hoang, T. H. T., A. D. Van, and H. T. T. Nguyen. 2017. Driving Variables for Eutrophication in Lakes of Hanoi by DataDriven Technique. Water and Environment Journal 31:176-83. https://doi.org/10.1111/wej.12231.

INEI. 2018. Resultados definitivos de los censos nacionales 20117: Amazonas. Instituto Nacional de Estadística e Informática, Lima, Lima, Perú.

Jeffrey, S. W., and G. F. Humphrey. 1975. New spectrophotometric equations for determining chlorophylls a, b, c1 and c2 in higher plants, algae and natural phytoplankton. Biochemie und Physiologie der Pflanzen 167:191-94. https: //doi.org/10.1016/S0015-3796(17)30778-3.

Kozak, A., A. Budzyńska, R. Dondajewska-Pielka, K. Kowalczewska-Madura, and R. Gołdyn. 2020. Functional groups of phytoplankton and their relationship with environmental factors in the restored Uzarzewskie Lake. Water (Switzerland) 
12:1-14. https://doi.org/10.3390/w12020313.

Lagomarsino, L., N. Diovisalvi, J. Bustingorry, R. Escaray, and H. E. Zagarese. 2014. Diel Patterns of Total Suspended Solids, Turbidity, and Water Transparency in a Highly Turbid, Shallow Lake (Laguna Chascomús, Argentina). Hydrobiologia 752:21-31. https://doi.org/10.1007/s10750-014-2013-3.

López-Martínez, M. L., G. A. Jurado-Rosero, I. D. Páez-Montero, and S. M. Madroñero-Palacios. 2017. Estructura térmica del lago Guamués, un lago tropical de alta montaña. Luna Azul 44:94-119. https://doi.org/10.17151/luaz.2017.44.7.

López-Martínez, M. L., and S. M. Madroñero-Palacios. 2015. Estado trófico de un lago tropical de alta montaña: Caso laguna de La Cocha. Ciencias e Ingeniería Neogranadina 25:21-42. https://doi.org/10.18359/rcin.1430.

Michelutti, N., J. L. Lemmen, C. A. Cooke, W. O. Hobbs, A. P. Wolfe, J. Kurek, and J. P. Smol. 2016. Assessing the effects of climate and volcanism on diatom and chironomid assemblages in an Andean lake near Quito, Ecuador. Journal of Limnology 75:275-286. https://doi.org/10.4081/jlimnol.2015.1323

MAGRAMA. 2013a. Protocolo de muestreo de fitoplancton en lagos y embalses. M-LE-FP-2013. Ministerio de Agricultura, Alimentación y Medio Ambiente, Madrid, Madrid, España.

MAGRAMA. 2013b. Protocolo de análisis y cálculo de métricas de fitoplancton en lagos y embalses. MFIT-2013. Ministerio de Agricultura, Alimentación y Medio Ambiente, Madrid, Madrid, España.

MINAGRI. 2013. Capitalizando los saberes locales. Ministerio de Agricultura y Riego, Lima, Lima, Perú.

Naeher, S., R. H. Smittenberg, A. Gilli, E. P. Kirilova, A. F. Lotter, and C. J. Schubert. 2012. Impact of recent lake eutrophication on microbial community changes as revealed by high resolution lipid biomarkers in Rotsee (Switzerland). Organic Geochemistry 49:86-95. https://doi.org/10.1016/j.orggeochem.2012.05.014.

Ni, M., J. L. Yuan, M. L., and Z. M. Gu. 2018. Assessment of water quality and phytoplankton community of Limpenaeus vannamei pond in intertidal zone of Hangzhou Bay, China. Aquaculture Reports 11:53-58. https://doi.org/10.1016/ j.aqrep.2018.06.002.

O'Neill, S. W., and A. P. Davis. 2012. Water treatment residual as a bioretention amendment for phosphorus. II: Longterm column studies. Journal of Environmental Engineering 138:328-36. https://doi.org/10.1061/(ASCE)EE.19437870.0000436.

Oliva, M., C. Oliva, D. Rojas, M. Oliva, and A. Morales. 2015a. Identificación botánica de especies nativas de pastos más importantes de las cuencas lecheras de Molinopampa, Pomacochas y Leymebamba, Amazonas, Perú. Scientia Agropecuaria 6:125-29. https://doi.org/10.17268/sci.agropecu.2015.02.05.

Oliva, M., D. Rojas, A. Morales, C. Oliva, and M. A. Oliva. 2015b. Contenido nutricional, digestibilidad y rendimiento de biomasa de pastos nativos que predominan en las cuencas ganaderas de Molinopampa, Pomacochas y Leymebamba, Amazonas, Perú. Scientia Agropecuaria 6:211-15. https://doi.org/10.17268/sci.agropecu.2015.03.07.

Pérez, A., W. Machado, D. Gutiérrez, A. C. Borges, S. R. Patchineelam, and C. J. Sanders. 2018. Carbon accumulation and storage capacity in mangrove sediments three decades after deforestation within a eutrophic Bay. Marine Pollution Bulletin 126:275-80. https://doi.org/10.1016/j.marpolbul.2017.11.018.

Primpas, J., and M. Karydis. 2011. Scaling the Trophic Index (TRIX) in Oligotrophic Marine Environments. Environmental Monitoring and Assessment 178:257-69. https://doi.org/10.1007/s10661-010-1687-x.

Pulido-López, P. C., and G. A. Pinilla-Agudelo. 2017. Evaluación del estado trófico de El Salitre, último humedal urbano de referencia en Bogotá. Revista de la Academia Colombiana de Ciencias Exactas, Físicas y Naturales 41:41-50. https: //doi.org/10.18257/raccefyn.411

Qin, B. Q., G. Gao, G. W. Zhu, Y. L. Zhang, Y. Z. Song, X. M. Tang, H. Xu, and J. M. Deng. 2013. Lake Eutrophication and its ecosystem response. Chinese Science Bulletin 58:961-70. https://doi.org/10.1007/s11434-012-5560-x.

Rahman, M. M., and H. Hamidah. 2020. Water quality influence the phytoplankton and bacteria abundance comparison between shallow freshwater and saltwater ponds. Desalination and Water Treatment 188:436-443. https://doi.org/ 10.5004/dwt.2020.25306.

Ramírez, R., C. Paredes, and J. Arenas. 2003. Moluscos del Perú. Revista de Biología Tropical 51:225-84. https://oi.org/ 10.15517/rbt.v51i3.26386

Rascón, J., W. Gosgot Angeles, M. Oliva, L. Quiñones, and M. Á. Barrena Gurbillón. 2020. Determinación de las épocas lluviosas y secas en la ciudad de Chachapoyas para el periodo de 2014-2018. Revista de Climatología 20:15-28.

Reichwaldt, E. S., and A. Ghadouani. 2012. Effects of rainfall patterns on toxic cyanobacterial blooms in a changing climate: Between simplistic scenarios and complex dynamics. Water Research 46:1372-93. https://doi.org/10.1016/ j.watres.2011.11.052.

Ren, Z., X. Qu, W. Peng, Y. Yu, and M. Zhang. 2019. Functional properties of bacterial communities in water and sediment of the eutrophic river-lake system of Poyang Lake, China. PeerJ 7:e7318. https://doi.org/10.7717/peerj.7318.

Rull, V., J. A. López-Sáez, and T. Vegas-Vilarrúbia. 2008. Contribution of non-pollen palynomorphs to the paleolimnological study of a high-altitude Andean lake (Laguna Verde Alta, Venezuela). Journal of Paleolimnology 40:399-411. https://doi.org/10.1007/s10933-007-9169-z

Santos, J. C. N., E. M. De Andrade, J. R. Araújo Neto, A. C. Maia Meireles, and H. A. Q. Palácio. 2014. Land use and trophic state dynamics in a tropical semi-arid reservoir. Revista Ciência Agronômica 45:35-44. https://doi.org/ 10.1590/S1806-66902014000100005.

Schindler, D. W., R. E. Hecky, and G. K. Mccullough. 2012. The rapid eutrophication of lake Winnipeg: Greening under Global Change. Journal of Great Lakes Research 38:6-13. https://doi.org/10.1016/j.jglr.2012.04.003.

Schoenebeck, C. W., M. L. Brown, S. R. Chipps, and D. R. German. 2012. Nutrient and algal responses to winterkilled fish-derived nutrient subsidies in eutrophic lakes. Lake and Reservoir Management 28:189-99. https://doi.org/10.1080/ 
07438141.2012 .693574$.

Scordo, F., C. V. Spetter, C. Seitz, M. C. Piccolo, and G. M. E. Perillo. 2020. Spatial and seasonal dynamics of water physical-chemical parameters in rivers and lakes of an Argentinian Patagonia basin. Environmental Earth Sciences 79:1-19. https://doi.org/10.1007/s12665-020-09063-7

Silvino, R. F., and F. A. R. Barbosa. 2015. Eutrophication potential of lakes: An integrated analysis of trophic state, morphometry, land occupation, and land use. Brazilian Journal of Biology 75:607-15. https://doi.org/10.1590/15196984.18913.

Van Colen, W., K. Portilla, T. Oña, G. Wyseure, P. Goethals, E. Velarde, and K. Muylaert. 2017. Limnology of the Neotropical High Elevation Shallow Lake Yahuarcocha (Ecuador) and Challenges for Managing Eutrophication Using Biomanipulation. Limnologica 67:37-44. https://doi.org/10.1016/j.limno.2017.07.008.

Venturoti, G. P., A. C. Veronez, R. V. Salla, and L. C. Gomes. 2015. Variation of limnological parameters in a tropical lake used for tilapia cage farming. Aquaculture Reports 2:152-57. https://doi.org/10.1016/j.aqrep.2015.09.006.

Vollenweider, R. A., F. Giovanardi, G. Montanari, and A. Rinaldi. 1998. Characterization of the trophic conditions of marine coastal eaters with special reference to the NW Adriatic Sea: Proposal for a trophic scale, turbidity and generalized water quality index. Environmetrics 9:329-57. https://doi.org/10.1002/(SICI)1099-095X(199805/06)9:3<329: :AID-ENV308>3.0.CO;2-9.

Wang, S., J. Li, B. Zhang, E. Spyrakos, A. N. Tyler, Q. Shen, F. Zhang, T. Kusterd, M. K. Lehmanne, Y. Wua, and D. Peng. 2018. Trophic State Assessment of Global Inland Waters Using a MODIS-Derived Forel-Ule Index. Remote Sensing of Environment 217:444-60. https://doi.org/10.1016/j.rse.2018.08.026.

Wetzel, R. G. 2001. Limnology: Lake and River Ecosystems. Academic Press Elservier, San Diego, California, USA.

Yao, L., C. Chen, G. Liu, and W. Liu. 2017. Science of the total environment sediment nitrogen cycling rates and microbial abundance along a submerged vegetation gradient in a eutrophic lake. Science of the Total Environment 616-617: 899-907. https://doi.org/10.1016/j.scitotenv.2017.10.230.

Zamparas, M., and I. Zacharias. 2014. Restoration of eutrophic freshwater by managing internal nutrient loads. A review. Science of the Total Environment 496:551-62. https://doi.org/10.1016/j.scitotenv.2014.07.076.

Zhao, X., Y. Zhou, J. Min, S. Wang, W. Shi, and G. Xing. 2012. Nitrogen runoff dominates water nitrogen pollution from rice-wheat rotation in the Taihu lake Region of China. Agriculture, Ecosystems and Environment 156:1-11. https://doi.org/10.1016/j.agee.2012.04.024.

Zheng, Z. C., T. X. Li, F. F. Zeng, X. Z. Zhang, H. Y. Yu, Y. D. Wang, and T. Liu. 2013. Accumulation characteristics of and removal of nitrogen and phosphorus from livestock wastewater by Polygonum hydropiper. Agricultural Water Management 117:19-25. https://doi.org/10.1016/j.agwat.2012.10.017. 